\title{
Antioxidant Potential of Pomegranate (Punica granatum L.) Cultivars Grown in Sri Lanka
}

\author{
Dilini Bopitiya and Terrence Madhujith ${ }^{{ }^{*}}$ \\ Postgraduate Institute of Science \\ University of Peradeniya \\ Sri Lanka
}

\begin{abstract}
The present study was carried out to determine the total phenolic content (TPC) and antioxidant potential of extracts obtained from aril of Sri Lankan cultivars Nayana, Daya, Nimali and Indian cultivar of pomegranate (Punica granatum L.). Total phenolic content was determined using Folin-Ciocalteu colorimetric method and expressed as $\mathrm{mg}$ gallic acid equivalents (GAE) per $L$ of extract. Antioxidant potential of the fruit extracts was measured using DPPH radical scavenging and FRAP assays and $\beta$ carotene/linoleate model system. The total antioxidant capacity (TAC) was measured using ABTS radical. The TPC varied widely among cultivars from $1199 \mathrm{mg} G A E / L$ of extract in Indian cultivar to $2390 \mathrm{mg}$ GAE/L of extract in Daya. The Daya cultivar possessed the highest TPC, followed by Nayana, Nimali, and Indian cultivars. Despite the moderate TPC, Nayana showed the highest antioxidant potential as determined by all four methods $\left(I_{50}\right.$ value of $0.182 \mathrm{mg} / \mathrm{mL}$, TAC of 93\%, FRAP of $6.33 \mathrm{~mol} \mathrm{Fe}(\mathrm{II}) / \mathrm{L}$ and $91 \%$ inhibition of oxidation of $\beta$-carotene). The TPC and antioxidant activity were not well correlated $(0.21 \leq \mathrm{r}$ $\leq 0.68$ ). The poor correlation may be attributable to the differential behaviour of phenolic constituents. Results revealed that the pomegranate can be categorized as a fruit with extremely high antioxidant potential.
\end{abstract}

Keywords: Antioxidants, $I C_{50}$ value, pomegranate (Punica granatum L.), total antioxidant capacity, total phenolic content

\section{INTRODUCTION}

Epidemiological studies indicate that frequent consumption of fruits and vegetables is associated with low risk of chronic diseases such as diabetes, cardiovascular diseases and cancers (Kriengask et al., 2006, Onder et al., 2009 and Safaa, et al., 2010). The increased intake of natural antioxidants, particularly the antioxidative compounds present in fruits and vegetables contributes to the antioxidant capacity of plasma and these constituents are reported to mitigate the damage caused by the oxidative stress (Lie et al., 2005, Oviasogie et al., 2009 and Vidhan et al., 2010). Recent studies reveal that the defensive effect of fruits and vegetables is at least partially attributable to the phytochemicals such as vitamins A, C and E, flavonoids, phenolic acids, lignans and carotenoids (Marja et al., 1999, Namjooyan et al., 2010 and Vinay et al., 2010). These constituents serve as free radical scavengers, hydrogen donating compounds, singlet oxygen quenchers and metal chelators. Therefore, it

\footnotetext{
Department of Food Science and Technology, Faculty of Agriculture, University of Peradeniya, Peradeniya, Sri Lanka

* $\quad$ Author for correspondence: madujith@yahoo.com
} 
is imperative to evaluate the commonly consumed fruits and vegetables for their antioxidative efficacy.

Pomegranate (Punica granatum L.) is considered as a medicinal and nutritional fruit due mainly to its antioxidant, antitumor, antihepatotoxic, antilipoperoxidative and antibacterial properties. It is popularly consumed as a fresh fruit, a beverage (juice and wine) and in processed forms such as jam and jelly (Wenjuan et al., 2010 and Vidhan et al., 2010). Research work carried out using animal and human models indicate that pomegranate juice has strong antioxidant activity, which is attributable to a diverse group of polyphenols including ellagitannins, gallotannins, ellagic acid, and flavonoids such as anthocyanins (Maria et al., 2000, Wenjuan et al., 2010, and Vidhan et al., 2010). Furthermore, some studies have exhibited that pomegranate possesses strong antioxidant activity and polyphenolic content superior to those of red wine, grapes, cranberry, orange, and apple while equal to or better than that of green tea (Maria et al., 2000, Kriengask et al., 2006 and Navindra et al., 2008).

More detailed information on health promoting components of pomegranate may provide a better insight into the neutraceutical, pharmaceutical and medicinal values of pomegranate which will invariably increase the consumption of pomegranate by the general public. The antioxidant activity of pomegranate juice has been found to vary considerably depending upon the cultivar, geoclimatic factors, harvesting, processing, and storage conditions. However, detailed investigations of the antioxidant activity of pomegranate varieties cultivated in Sri Lanka are very limited. In this backdrop, the objective of this study was to determine the total phenolic content and antioxidant capacity of most abundant pomegranate cultivars namely, Nayana, Daya, Nimali, and to compare the activities of Sri Lankan cultivars with a common Indian cultivar.

\section{METHODOLOGY}

2,2-diphenyl-1-picrylhydrazyl hydrate (DPPH), Folin-Ciocalteu's phenol reagent, gallic acid, 2,2'-azinobis (3-ethylbenzothiazoline-6-sulfonic acid) diammonium salt (ABTS), linoleic acid, 2, 4, 6-tris(2-pyridyl)-1,3,5-triazine (TPTZ), $\beta$-carotene, and Tween 20 were purchased from Sigma chemicals company (MO,USA). All other chemicals used were of analytical grade and obtained from either Sigma Chemicals or Himedia.

Matured healthy pomegranate fruits (with firm texture and colour fully developed) of three cultivars, namely Nayana, Daya, and Nimali were obtained from the Regional Research Station of Department of Agriculture, Makandura, Sri Lanka. Fruits belonging to a commonly available Indian cultivar were purchased from the local market. Fig. 1 illustrates the external features of the four cultivars of pomegranate used for the study. 


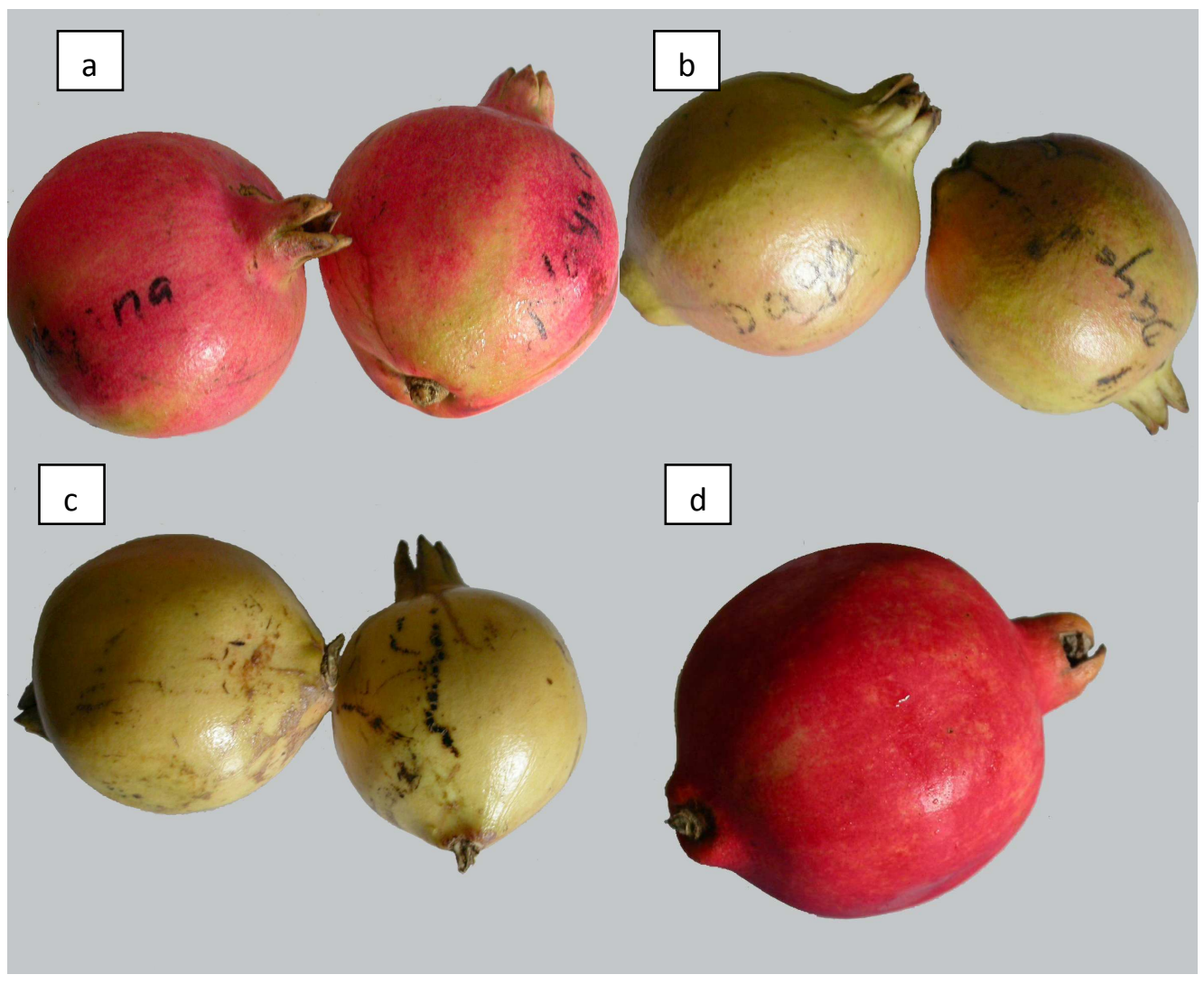

Fig. 1. External features of (a) Nayana (b) Daya (c) Nimali and (d) Indian pomegranate cultivars used for the study.

\section{Preparation of aril extract}

Pomegranate fruits were washed with pure water, peeled and edible portion (arils) was carefully separated. The so separated arils were manually pressed to obtain fruit extract, which was then filtered through a cotton mesh and subsequently through a filter paper (Whatman No. 01) and the resulting extracts were stored at $-20^{\circ} \mathrm{C}$ until further analysis. The extracts were appropriately diluted with distilled water and used for chemical analysis.

\section{Determination of total phenolic content (TPC)}

The total phenolic content (TPC) of the extracts was determined colorimetrically using Folin-Ciocalteu's reagent as described by Kriengsak et al. (2006) with some minor modifications. Fruit extracts $(20 \mu \mathrm{L})$ were mixed with $100 \mu \mathrm{L}$ of Folin-Ciocalteu's reagent, left for 3 minutes and subsequently $300 \mu \mathrm{L}$ of sodium carbonate $(0.7 \mathrm{M})$ was added and vortexed. The absorbance of the resulting mixture was measured at $725 \mathrm{~nm}$ using a UV visible spectrophotometer (UV 1601, Shimadzu, Japan) after leaving for 30 minutes at the room temperature $\left(25^{\circ} \mathrm{C}\right)$. The results were expressed as $\mathrm{mg}$ gallic acid equivalents (GAE) per litre of extract using a gallic acid $(50-500 \mathrm{mg} / \mathrm{L})$ standard curve. 


\section{Determination of DPPH radical scavenging capacity}

Antioxidant capacity of the fruit extracts was assessed using 2,2-diphenyl-1picrylhydrazylhydrate (DPPH). The DPPH radical scavenging activity of pomegranate extracts was quantified according to the method reported by Kai Marxen et al., (2007) with minor modifications.

For DPPH radical scavenging assay, five different concentrations ranging between 0.1 and $1.0 \mathrm{mg} / \mathrm{mL}$ of each extract $(0.5 \mathrm{~mL})$ was mixed with $2.5 \mathrm{~mL}$ of methanolic $\mathrm{DPPH}$ radical $(0.1 \mathrm{mM})$. After leaving for 20 minutes in the dark at room temperature, the absorbance was recorded at $517 \mathrm{~nm}$ using a UV visible spectrophotometer (UV 1601, Shimadzu, Japan). The radical scavenging activity (RSA) was calculated as percentage DPPH discoloration using the following equation.

$$
\operatorname{RSA}(\%)=\left[\left(\mathrm{A}_{\mathrm{DPPH}}-\mathrm{A}_{\text {Sample }}\right) / \mathrm{A}_{\mathrm{DPPH}}\right] \times 100
$$

where, $\mathrm{A}_{\text {Sample }}$ is the absorbance of the solution containing the extract after 20 minutes and A DPPH is the absorbance of the DPPH solution devoid of extract. Results were expressed as $\mathrm{IC}_{50}$ value that denotes the concentration of the sample required to scavenge $50 \%$ of DPPH radicals.

\section{Determination of ABTS radical scavenging capacity}

The total antioxidant capacity of the extracts was determined using ABTS radical. The $\mathrm{ABTS}^{*+}$ was generated by reacting 2,2'-azobis (2-ethylbenzothiazoline-6-sulfonic acid) diammonium salt (ABTS) with 2,2'-azobis (2-methylpropanimidamide) dihydrochloride $(\mathrm{AAPH})$, which acts as the radical generator. Each extract $(40 \mu \mathrm{L})$ was mixed with $1.96 \mathrm{~mL}$ of ABTS radical solution and absorbance was measured over 6 minutes at 1 minute interval at $765 \mathrm{~nm}$ using a UV visible spectrophotometer (UV 1601, Shimadzu, Japan). The radical scavenging activity (RSA) after lapse of 1 minute was calculated as percentage of ABTS $^{++}$ discoloration (Robereta et al., 1999).

\section{Determination of ferric reducing antioxidant power (FRAP)}

Ferric reducing antioxidant power (FRAP) assay was performed according to the method explained by Kriengask et al. (2006) with some modifications. The FRAP reagent included $10 \mathrm{mM} \mathrm{2,} \mathrm{4,} \mathrm{6-tris} \mathrm{(2-pyridyl)-1,} \mathrm{3,} \mathrm{5-triazine} \mathrm{(TPTZ),} 20 \mathrm{mM} \mathrm{FeCl}_{3}$, and $0.3 \mathrm{M}$ acetate buffer at 1:1:10 (v/v) ratios. Three millilitres of FRAP reagent at $37^{\circ} \mathrm{C}$ were mixed with 50 $\mu \mathrm{L}$ of the extract. After a lapse of 4 minutes, the absorbance was read at $593 \mathrm{~nm}$ against a reagent blank. The results were expressed as mol of Fe (II) per litre of extract using a Fe (II) $(0.2-1.0 \mathrm{M})$ standard curve.

\section{Determination of antioxidant efficacy using $\beta$-carotene/linoleate model system}

The ability of pomegranate extracts to inhibit $\beta$-carotene bleaching was estimated according to the method explained by Maria et al. (2010) with minor modifications. $\beta$ Carotene/linoleate emulsion was prepared by mixing $10 \mathrm{mg}$ of $\beta$-carotene, $40 \mathrm{mg}$ of linoleic acid, $600 \mathrm{mg}$ of Tween 20 emulsifier and $100 \mathrm{~mL}$ of oxygenated deionized water. The so prepared emulsion $(2 \mathrm{~mL})$ was mixed with $200 \mu \mathrm{L}$ of extracts and incubated at $50^{\circ} \mathrm{C}$. The oxidative loss of emulsion was monitored using a UV visible spectrophotometer (UV 1601, Shimadzu, Japan) at $470 \mathrm{~nm}$ wave length at every 15 minute for 120 minutes. The 
antioxidant activity (AA \%) was calculated according to the following equation and expressed as percentage value:

$$
\mathrm{AA}(\%)=\left\{\left[\left(\mathrm{AC}_{120}-\mathrm{AC}_{0}\right)-\left(\mathrm{AA}_{120}-\mathrm{AA}_{0}\right)\right] /\left(\mathrm{AC}_{120}-\mathrm{AC}_{0}\right)\right\} \times 100
$$

where, $\mathrm{AA}_{120}$ is the absorbance of the antioxidant at 120 minutes, $\mathrm{AC}_{120}$ is the absorbance of the control at 120 minutes, $\mathrm{AA}_{0}$ is the absorbance of the antioxidant at 0 minute, and $\mathrm{AC}_{0}$ is the absorbance of the control at 0 minute.

\section{Analysis of data}

All experiments were conducted in triplicates and completely randomized design was used. The statistical analysis was carried out using MS Office (Excel) and MINITAB version 16 software.

\section{RESULTS AND DISCUSSION}

\section{Total Phenolic Content}

Phenolic constituents are one of the major group of compounds serving as primary antioxidants, especially as free radical terminators (Marja et al., 1999 and Oviasogie et al., 2009). The total phenolic content of the selected pomegranate fruit extracts tested is illustrated in Fig 2. The Total phenolic content varied widely among the cultivars tested from $1199 \mathrm{mg} \mathrm{GAE} / \mathrm{L}$ (Indian cultivar) to $2390 \mathrm{mg} \mathrm{GAE} / \mathrm{L}$ (Daya cultivar). The Daya cultivar possessed the highest TPC followed by Nayana, Nimali, and Indian cultivars. These results confirm that the amount of TPC is cultivar-dependant. Similar results have been reported in fruits such as strawberry, guava and blueberry (Kriengask et al., 2006 and Onder et al., 2009).

Based on the TPC, pomegranate aril juice can be identified as a fruit with potential antioxidant capacity. Fruits that show TPC of $1-5 \mathrm{~g}$ GAE/L are considered fruits with medium TPC (Maria do et al., 2010). Pomegranate contained high TPC compared to most of the commonly available fruits. The TPC of pineapple, mango, papaya, apple, and berries are 47.9, 56.0, 57.6, 11.9, and $28.7 \mathrm{mg} / 100 \mathrm{~g}$, respectively (Kriengsak et al., 2006). Moreover, pomegranate is superior even to green tea $(1029 \mathrm{mg} \mathrm{GAE} / \mathrm{L})$ and equal or better to red wine (2036 mg GAE/L) (Maria do et al., 2010). Maria et al. (2000) have also reported similar findings indicating that fresh pomegranate from Africa contains $1800-2100 \mathrm{mg}$ GAE/L. Further, the results of the present study tally with that of Mariela et al. (2010) which reported TPC of $1303-2270 \mathrm{mg} \mathrm{GAE} / \mathrm{L}$. 


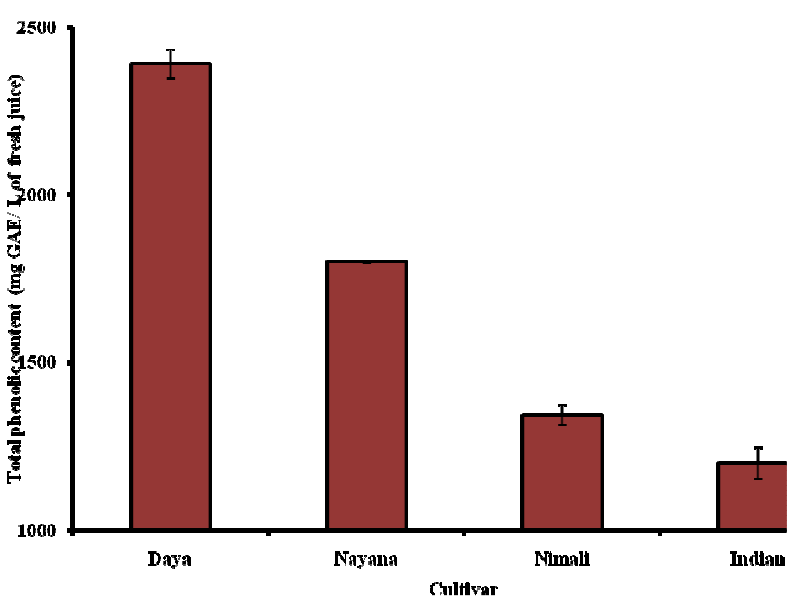

Fig. 2. Total Phenolic Content (TPC) of pomegranate extracts.

\section{DPPH radical scavenging activity and total antioxidant capacity}

DPPH and ABTS radical scavenging activities of the tested extracts are summarized in Table1. The DPPH radical scavenging activity was expressed as $\mathrm{IC}_{50}$ value which is inversely proportional to the antioxidant activity (Kriengask et al., 2006 and Onder et al., 2009). Results shown in Table1 indicate that the $\mathrm{IC}_{50}$ value of pomegranate extracts ranged between $0.182 \mathrm{mg} / \mathrm{mL}$ to $0.446 \mathrm{mg} / \mathrm{mL}$ with Nayana cultivar showing the highest antioxidant activity followed by the Indian cultivar, Nimali, and Daya.

Fruits showing an $\mathrm{IC}_{50}$ value of less than $1 \mathrm{mg} / \mathrm{mL}$ is categorized as fruits with extremely high antioxidant potential (Safaa et al., 2010). According to the $\mathrm{IC}_{50}$ value, pomegranate can be categorized as a fruit with high antioxidant potential. The antioxidant activity measured by DPPH assay was comparable to that of red grapes $0.43 \mathrm{mg} / \mathrm{mL}$ which contained an exceptional antioxidant activity (Kriengsak et al., 2006). Therefore, pomegranate can be considered a fruit that has an exceptionally high antioxidant activity. Furthermore, Kriengsak et al. (2006) reported that the $\mathrm{IC}_{50}$ value of selected fresh fruits, namely banana, tomato, orange, apple, dates, strawberry, kiwi, and pear range from $1.65-15.93 \mathrm{mg} / \mathrm{mL}$. Safaa et al. (2010) also reported similar results for pomegranate $(0.53 \mathrm{mg} / \mathrm{mL})$ and results of these studies support the findings of the present study.

Table 1. DPPH and ABTS radical scavenging activity of pomegranate extracts

\begin{tabular}{lcc}
\hline Cultivar & $\begin{array}{c}\mathbf{I C}_{\mathbf{5 0}} \text { value } \\
(\mathrm{mg} / \mathrm{mL})\end{array}$ & $\begin{array}{c}\text { ABTS } \\
(\%)\end{array}$ \\
\hline Daya & $0.446 \pm 0.05^{\mathrm{c}}$ & $72.73 \pm 1.9^{\mathrm{f}}$ \\
Nayana & $0.182 \pm 0.02^{\mathrm{b}}$ & $93.1 \pm 2.0^{\mathrm{d}}$ \\
Nimali & $0.368 \pm 0.05^{\mathrm{a}}$ & $91.2 \pm 1.5^{\mathrm{d}}$ \\
Indian & $0.362 \pm 0.04^{\mathrm{a}}$ & $89.7 \pm 2.03^{\mathrm{e}}$ \\
\hline
\end{tabular}

\pm SD values with different superscripts are significantly different $(\mathrm{p} \leq 0.05)$

Total antioxidant capacity of pomegranate extracts as measured by ABTS radical varied between $93.1 \%$ and $72.73 \%$ with the highest being observed in Nayana cultivar (Table 1). The reaction between the extracts and $\mathrm{ABTS}^{*+}$ was almost completed within the first minute 
of the reaction (0.60 to 0.059$)$ (Fig. 3). The extract of Nayana cultivar was the most active (the absorbance after 6 minutes. was 0.010 ). The activity of Nayana, Nimali, and the Indian cultivar was clearly stronger than that of Daya (Table 1 and Fig. 3). Based on the fast scavenging activity, pomegranate extracts revealed an extremely high antioxidant activity. Fruits at $10 \%$ level showing an absorbance less than 0.5 after 1 minute of reaction time are categorized as fruits with extremely high antioxidant capacity (Zhang et al., 2009).

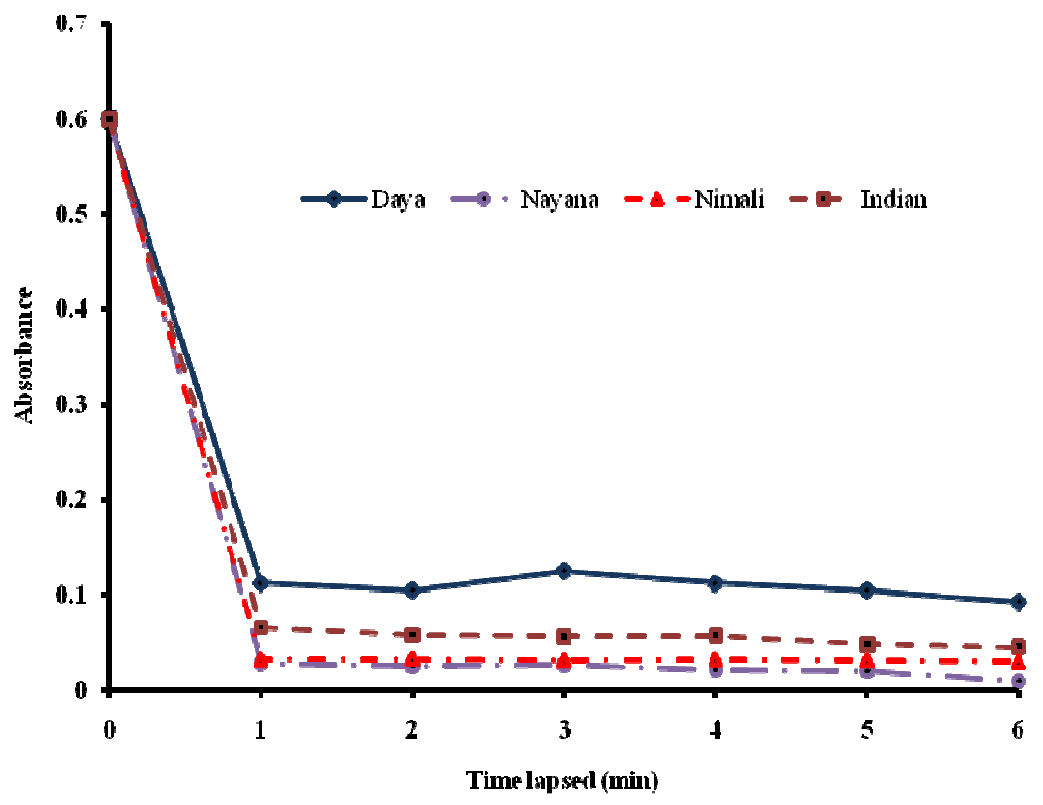

Fig. 3. Reduction of the concentration of $\mathrm{ABTS}^{\circ+}$ reflected by the reduction of absorbance in the presence of different pomegranate extracts over six minutes.

\section{Ferric reducing antioxidant power (FRAP)}

Ferric reducing antioxidant power (FRAP) assay is based on the reduction of $\mathrm{Fe}^{+3}$-TPTZ complex into $\mathrm{Fe}^{+2}$-TPTZ form in the presence of antioxidants (Kriengsak et al., 2006). It quantifies the reducing power of antioxidative extract which is an integral attribute of such compounds. FRAP values obtained from the regression equation of a calibration curve $(y=-$ $0.311 \mathrm{x}+0.986, \mathrm{R}^{2}=-0.89$ ) is illustrated in Fig. 4. The FRAP value of Indian and Nayana cultivars ranged from 2.12 to $6.33 \mathrm{~mol}$ of $\mathrm{Fe}$ (II) / L. The highest reducing power was observed in Nayana cultivar extract while the lowest effect was observed in Daya cultivar.

FRAP value of all pomegranate cultivars tested lied above $1000 \mu \mathrm{mol} \mathrm{Fe}$ (II)/g of extract. Thus, pomegranate can be categorized as fruit with high antioxidant activity (Vinay et al., 2010). Furthermore, pomegranate extracts showed high FRAP value compared to foods containing high level of antioxidant activity such as green tea and red wine (Maria et al., 2000). 


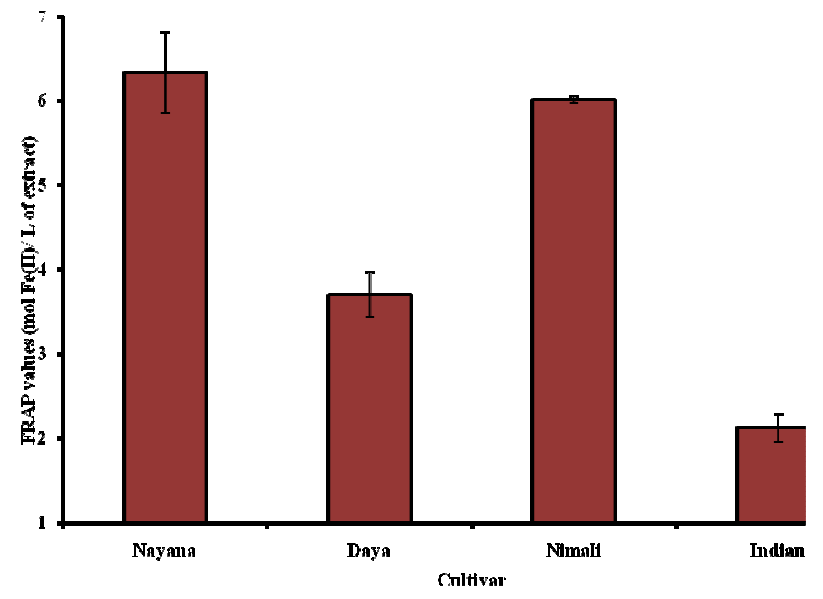

Fig. 4. FRAP values exhibited by different pomegranate extracts.

\section{$\beta$-Carotene-linoleate model system}

$\beta$-Carotene-linoleate model system is based on the inhibition of generation of volatile organic compounds, conjugated dienes, and hydroperoxides arising from linoleic acid oxidation (Onder et al., 2009). The antioxidant capacity of pomegranate extracts tested as measured by percentage of oxidation inhibition is illustrated in Fig. 5. The highest antioxidant capacity was observed in Nayana (91.02\%), followed by Nimali $(86.02 \%)$, Indian (84.88\%), and Daya (79.94\%) cultivars.

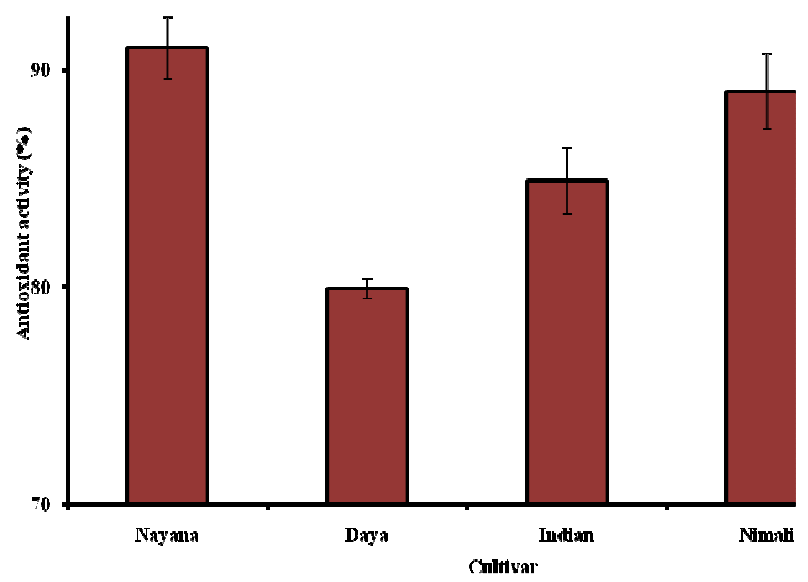

Fig. 5. The efficacy of different pomegranate extracts in inhibiting bleaching of $\beta$ carotene.

All pomegranate extracts tested effectively retarded the oxidation of $\beta$-carotene in the emulsion. Maria do et al. (2010) classified antioxidant capacity of fruits as high $(>70 \%)$, intermediate $(40-70 \%)$ or low $(<40 \%)$ levels depend on oxidation inhibition. Therefore, pomegranate can be categorized as a fruit with high antioxidant capacity.

Antioxidant activity as measured by ABTS, DPPH, FRAP assays and using $\beta$ carotene/linoleate model system were not well correlated with TPC $(0.21 \leq \mathrm{r} \leq 0.68)$. Poor 
correlation between TPC and these four parameters is reported by some authors worked on fruits. Kriengsak et al. (2006) reported no correlation between TPC and antioxidant capacity as determined by DPPH, FRAP, and ABTS assays in peaches, plums and nectarines. Moreover, Onder et al. (2009) also reported a poor correlation for jujube fruit.

Despite the moderate TPC, Nayana cultivar showed the highest antioxidant activity as measured by DPPH, ABTS, FRAP assays, and $\beta$-carotene/linoleic model system. On the other hand, Daya cultivar showed a relatively less antioxidant activity though it contains high level of TPC.

The poor correlation may be attributable to differential behaviour of phenolic constituents. The presence of other phytochemicals such as ascorbic acid, tocopherol and pigments, which also contribute to the total antioxidant capacity may also be attributable for the poor correlation.

Use of a single method to determine antioxidant capacity is insufficient, thus adoption of different assays and model systems provide a better insight into the actual activity of the extracts. The correlation among TAC, DPPH radical scavenging activity, FRAP and the protective activity as measured by $\beta$-carotene-linoleate model system was strong and ranged between 0.68 and 0.98 with the strongest correlation observed between DPPH radical scavenging activity and the efficacy as measured by the $\beta$-carotene-linoleate model system ( $\mathrm{r}$ $=0.96)$ while the lowest correlation was observed between TAC and FRAP assays $(r=0.68)$.

Based on the chemical assays carried out in this study, Nayana and Nimali cultivars exhibited strong antioxidant activity than the Indian cultivar while Daya showed a relatively lower activity.

\section{CONCLUSIONS}

Pomegranate can be categorized as a fruit with high antioxidant potential. Nayana cultivar possessed the strongest antioxidant activities followed by Nimali, Indian and Daya cultivars.

\section{ACKNOWLEDGMENTS}

The authors thank National Research Council (NRC) for their financial support and Regional Research Station of Agriculture, Makandura for providing samples.

\section{REFERENCES}

Artes, F., Tudela, J.A. and Gil, M.I. (1998). Improving the keeping quality of pomegranate fruit by intermittent warming. Eur. Food Res. Technol., 207, 316-32.

Giedrius, M. (2006). Screening, isolation and evaluation of antioxidative compounds from Geranium macrorrhizum, Potentilla fruticosa and Rhaponticum carthamoides. (Thesis) Wageningen University, Dutch and Lithuanian. 
Hala, M. Abdou. (2011). Comparative antioxidant activity study of some edible plants used spices in Egypt. Journal of American Science, 7(1).

Kai, M., Klaus, H.V., Sebastian, L., Ralf, H., Andreas, R. and Ulf-Peter, H. (2007). Determination of DPPH radical oxidation caused by methanolic extracts of some microalgal species by linear regression analysis of spectrophotometric measurements. Sensors, 7, 2080 2095.

Komes, D., Ana, B.C., Dunja, H., Gordana, R. and Marija, B. (2010). Phenolic compodition and antioxidant properties of some traditionally used medicinal plants affected by extraction time and hydrolysis. Journal of Phytochemical Analysis, 22, 172 - 180.

Kriengsak, T., Unaroj, B., Kevin, C., Luis, C. and David, H.B. (2006). Comparison of ABTS, DPPH, FRAP, and ORAC assays for estimating antioxidant activity from guava fruit extracts. Journal of Food Composition and Analysis, 19, 669 - 675.

Kruawan, K. and Kangsadalampal, K. (2006). Antioxidant activity, phenolic compound contents and antimutagenic activity of some water extract of herbs. Thai Journal of Pharm. Sci., 30, 28 - 35.

Labbe, M., Pena, A., and Saenz, C. (2010). Antioxidant capacity and phenolic composition of juice from pomegranate stored in refrigeration.International conference on food innovation (25 -29 October 2010), Universidad de Chile.

Lie-Fen, S., Jieh-Hen, T., Je-Hsin, C., Chih-Yang, C. and Chiu-Ping, L. (2005). Antioxidant properties of extracts from medicinal plants popularly used in Taiwan. International Journal of Applied Science and Engineering, 3(3), 195 - 202.

Ilhami, G., Raid, E., Akcahan, G., Laurent, B. and Ekrem, K. (2007).A comparative study on the antioxidant activity of fringe tree extracts. African Journal of Biotechnology, 6(4), 410 - 418.

Maria do S. M.R., Ricardo E.A., Edy, S. de B., Jara, P., Fulgencio, S. and Jorge, M. (2010). Bioactive compounds and antioxidant capacities of 18 non-traditional tropical fruits from Brazil.Journal of Food Chemistry, 121, 996 - 1002.

Maria, I.G., Francisco, A.T., Betty, H., Deirdre, M.H. and Adel, A.K. (2000). Antioxidant activity of pomegranate juice and its relationship with phenolic composition and processing. Journal of Agriculture Food Chemistry, 48, 4581 - 4589.

Marja, P.K., Anu, I.H., Heikki, J.V., Jussi-pekka, R., Kalevi, P., Tytti, S.K. and Marina, H. (1999). Antioxidant activity of plant extracts containing phenolic compounds. Journal of Agriculture Food Chemistry, 47, 3954 - 3962.

Namjooyan, F., Azemi, M.E. and Rahmanian, V.R. (2010). Investigation of antioxidant activity and total phenolic content of various fractions of aerial parts of Pimpinella barbata (DC) bioss. Jundishapur Journal of Natural Pharmaceutical Products, 5(1), 1 - 5.

Navindra, P.S., Michael, A., Yanjun, Z., Susanne, M.H., Lydia, F., Mark, D. and David, H. (2008). Comparison of antioxidant potency of commonly consumed polyphenol rich beverages in United sates. Journal of Agriculture Food Chemistry, 56, 1415 - 1422. 
Onder, K., Sezai, E., Memnune, S., Celi, T. and Sedat, S. (2009). Total phenolics and antioxidant activity of jujube genotypes selected from Turkey. African Journal of Biotechnology, 8(2), 303 - 307.

Oviasogie, P.O., Okoro, D. and Ndiokwere, C.L. (2009). Determination of total phenolic amounts of some edible fruits and vegetables. African Journal of Biotechnology, 8(12), 2819 -2820.

Pourmorad, F., Hosseinimehr, S.J. and Shahabimajd, N. (2006). Antioxidant activity, phenol and flavonoid contents of some selected Iranian medicinal plants. African Journal of Biotechnology, $5(11), 1142-1145$.

Rezaeizadeh, A., Zuki, A.B.Z., Abdollahi, M., Goh, Y.M., Noordin, M.M., Hamid, M. and Azmi, T.I. (2011). Determination of antioxidant activity in methanolic and chloroformic extracts of Mormordica charantia. African Journal of Biotechnology, 10(24), 4932 - 4940.

Robereta, R., Nicoletta, P., Anna, P., Ananth, P., Min, Y. and Catherine, R. (1999). Antioxidant activity applying an improved ABTS radical cation decoloration assay. Journal of Free Radical Biology and Medicine, 26, (9/10), 1231.-.1237.

Safaa Y. Qusti, Ahmed N. Abo-khatwa, and Mona A. Bin Lahwa (2010). Screening of antioxidant activity and phenolic content of selected food items cited in the Holly Quran. European Journal of Biological Sciences (EJBS), 2 (1).

Vidhan, J., Ara DerMarderosian and John, R.P. (2010). Anthocyanins and polyphenol oxidase from dried arils of pomegranate (Punica granatum L.). Journal of Food Chemistry, 118, 11-16.

Vinay, R.P., Prakash, R.P. and Sushil, S.K. (2010). Antioxidant activity of some selected medicinal plants in weatern region of India. Advances in biological research, 4(1), 23 - 26.

Wenjuan, Q., Andrew, P.B., Zhongli, P. and Haile, Ma. (2012). Quantitative determination of major polyphenol constituents. Journal of Food Chemistry, 132, 1585-1591.

Zhang, W., Lin, B., Han, L. and Zhang, H. (2009). Antioxidant activities of extracts from areca flower, husk, and seed. African Journal of Biotechnology, 8(16), 3887 - 3892 